\title{
Silicon-a new substrate for GaN growth
}

\author{
S PAL and C JACOB* \\ Materials Science Centre, Indian Institute of Technology, Kharagpur 721 302, India
}

MS received 23 February 2004; revised 30 September 2004

\begin{abstract}
Generally, GaN-based devices are grown on silicon carbide or sapphire substrates. But these substrates are costly and insulating in nature and also are not available in large diameter. Silicon can meet the requirements for a low cost and conducting substrate and will enable integration of optoelectronic or high power electronic devices with $\mathrm{Si}$ based electronics. But the main problem that hinders the rapid development of GaN devices based on silicon is the thermal mismatch of $\mathrm{GaN}$ and $\mathrm{Si}$, which generates cracks. In 1998, the first MBE grown GaN based LED on Si was made and now the quality of material grown on silicon is comparable to that on sapphire substrate. It is only a question of time before $\mathrm{Si}$ based GaN devices appear on the market. This article is a review of the latest developments in GaN based devices on silicon.
\end{abstract}

Keywords. GaN; MOCVD; epitaxial growth; devices.

\section{Introduction}

Silicon substrates are low cost, available in large diameters and have well characterized electrical and thermal properties. Despite these advantages silicon has not been popular as a substrate material for $\mathrm{GaN}$ growth due to several problems mainly related to the cracking of $\mathrm{GaN}$ film due to stress. These stresses are not so high in magnitude for sapphire or $\mathrm{SiC}$ substrates. In 1971, group III nitrides (AIN) were grown on silicon by MOVPE (Manasevit et al 1971). However, research activity in group III nitrides remained stagnant over the next two decades until progress in GaN growth on sapphire by low temperature seed or buffer layer (Amano et al 1988) as well as the development of $p$-type doping technique (Amano et al 1989; Nakamura et al 1992) took place. Much of this phase of research centred around growth on sapphire and $\mathrm{SiC}$.

Silicon attracted attention as a substrate material for $\mathrm{GaN}$ growth when the first MBE grown GaN LED on Si was demonstrated in 1998 (Guha and Bojarczuk 1998a,b). This work demonstrated that $p$-type doping was achievable in $\mathrm{GaN}$ on Si too, and devices could be made out of it. Nevertheless, till recently, the properties of $\mathrm{GaN}$ on $\mathrm{Si}$ were rather poor and the FWHM of X-ray rocking curve of $\mathrm{GaN}$ on $\mathrm{Si}$ was 1000 arc s whereas for $\mathrm{GaN}$ on $\mathrm{SiC}$ it was 250-300 arc s. Photoluminescence spectra of the films grown on $\mathrm{Si}$ also show peak broadening. However, during the last couple of years, intensive research activities have been carried out in this area and at present the best $\mathrm{GaN}$ layers on $\mathrm{Si}$ are almost comparable to $\mathrm{GaN}$ layers on $\mathrm{SiC}$.

\footnotetext{
*Author for correspondence
}

\section{Problems associated with $\mathrm{Si}$}

The lattice mismatch between $\mathrm{Si}$ and $\mathrm{GaN}$ is almost $16 \%$ that causes a high dislocation density in the GaN layers but the major problem is the thermal mismatch, which is $54 \%$. Therefore, thick epilayers of $\mathrm{GaN}$ for device fabrication are not achievable without cracks.

Furthermore $\mathrm{Si}$ is available with resistivity up to $10^{4} \mathrm{ohm}$ $\mathrm{cm}$ that is much less than the resistivity value of sapphire, $\mathrm{SiC}$ or $\mathrm{GaN}$, and this may lead to parasitic capacitance effects during high frequency operation.

There are also several reports (Hashimoto et al 1997; Marchand et al 1999) of melt back etching of Si during $\mathrm{GaN}$ growth. Under $\mathrm{H}_{2}$ gas flow, at a temperature of around $1200 \mathrm{~K}$, Si substrate out-gases Si and, during growth, this Si will lead to a high $n$-type background doping making it difficult to achieve $p$-type doping. However, by using $\mathrm{NH}_{3}$ pre-treatment of $\mathrm{Si}$ wafer before growth of $\mathrm{GaN}$ layer, this effect can be reduced. Nitridation of $\mathrm{Si}$ is selflimiting and the nitridation process will create a thin layer (few nano meters) of $\mathrm{Si}_{x} \mathrm{~N}_{y}$ layer and will oppose the Si out-diffusion during growth.

\section{Different approaches for growth}

(I) GaN growth on sapphire, $\mathrm{SiC}$ or $\mathrm{Si}$ uses a seed layer or buffer layer to accommodate the lattice mismatch between the substrate and the epilayer. The effect of surface pre-treatment by exposure to $\mathrm{NH}_{3}$ has been studied for $6 \mathrm{H}-\mathrm{SiC}, 4 \mathrm{H}-\mathrm{SiC}$ and $3 \mathrm{C}-\mathrm{SiC}$ substrates for $\mathrm{GaN}$ growth (Lee et al 2000). After nitridation of SiC, the deposited $\mathrm{GaN}$ film was found to be polycrystalline. In case of pre-adsorption of TMG, epitaxial but island-like GaN formed on the substrate. In the third case, with an ultra- 
thin $(\sim 1.5 \mathrm{~nm})$ coverage of $\mathrm{AlN}$ on $\mathrm{SiC}$ (by preadsorption of TMA or by $50 \mathrm{~s}$ deposition of AIN), GaN epilayers were successfully deposited on SiC. However, for growth of GaN on Si substrates, a 20-30 nm thick AlN seed layer deposited well below the actual growth temperature of $\mathrm{GaN}$ on the substrates yields epitaxial quality GaN. Occasionally, before AlN predeposition, a few monolayers of $\mathrm{Al}$ are deposited over the substrate, which act as barrier for the nitridation of the Si substrate (Dadgar et al 2000a). Chen et al (2001) performed an extensive study on this topic and they showed that an optimum time of AlN predeposition leads to a steplike surface, deposition for a shorter time produces island growth of AIN and too long a growth time will end in a roughened surface of AlN. The surface roughening is due to the alloying reaction between $\mathrm{Al}$ and $\mathrm{Si}$. The AlN predeposition for very short time may lead to nitridation of the Si substrate surface. Therefore, their novel idea was to protect the Si surface by depositing few monolayers of $\mathrm{Al}$, which would be converted into AlN when exposed to ammonia. The reason for depositing low temperature AIN seed layer is to minimize the decomposition of $\mathrm{GaN}$ deposits on the reactor wall, which will influence the AIN seed layer growth. Low series resistance is one of the key factors for devices but $\mathrm{AlN}$ is a high resistivity material. But at low temperatures, the growth of the seed layer will result in non-stoichiometric composition and also produces a high density of imperfections in the structure, thereby lowering the barrier between the Si/AlN interface. GaN LEDs on Si have been shown to have the lowest series resistance using a low temperature seed layer (Dadgar et al 2002a). Thus, either pre-treatment or AlN seed layer will produce a nitrided buffer. However, the AlN seed layer seems to be most effective solution to the problem of mismatch.

(II) The main difficulty in growing $\mathrm{GaN}$ on $\mathrm{Si}$ is the stress that develops during growth. Cracks occur even for epilayer thickness of about $1 \mu \mathrm{m}$. Therefore, to achieve GaN based devices, it is important that the strain be minimized. For optoelectronic devices, a thick layer with good electro-optical and structural properties is required and these are also the basic criterion for films for transistors. Also, to achieve high carrier mobility the interface should be defect free, abrupt and smooth. This is achievable only for epilayers well above $1 \mu \mathrm{m}$ in thickness. Stress has another influence on device processing. It produces curvature of the wafer and during contact lithography the curvature creates problems. Usually the curvature of $\mathrm{GaN}$ on $\mathrm{Si}$ is about $2 \mathrm{~m}$ (Dadgar et al 2000b; Feltin et al 2001a). In case of heteroepitaxy, growth starts with small nuclei, which grow and coalesce. At the coalescence boundaries tensile stress of the order of $0 \cdot 1-0 \cdot 2 \mathrm{GPa} / \mu \mathrm{m}$ is generated during $\mathrm{GaN}$ growth on sapphire (Amano et al 1998; Hearne et al 1999; Etzkorn and Clarke et al 2001) and this generates cracks in the epilayer for thicknesses $>5 \mu \mathrm{m}$ ! The stress increases during cooling and at room temperature it becomes $0 \cdot 8-0.9 \mathrm{GPa} / \mu \mathrm{m}$. During epilayer growth of $\mathrm{GaN}$ on $\mathrm{Si}$ compressive stresses are generated. Fu et al (2000) showed that the residual stress is dependent on the impurity concentration in the film and it was observed that doping could enhance the tensile stress in the epilayer (Romano et al 2000; Terao et al 2001). If the tensile stress is comparable to the compressive stress generated due to thermal mismatch, crack free thick GaN epitaxy on $\mathrm{Si}$ will be possible. It is estimated that $\mathrm{Si}$ doping conc. of $5 \times 10^{18}$ in $\mathrm{GaN}$ and $\mathrm{AlGaN}$ can generate $0.5 \mathrm{GPa} / \mu \mathrm{m}$ tensile stress (Terao et al 2001). Thus, an approach that combines doped layers and lower temperature growth can result in GaN films with low stresses.

(III) Substrates containing amorphous or gliding layers e.g. SIMOX or SOI, have great potential for use in GaN growth. Slipping of the crystal over the amorphous layer will effectively lower stresses that develop during growth. Steckl et al (1996) converted the thin top layer of Si (111) of a SOI substrate into $\mathrm{SiC}$ by carbonization. This was then used as a substrate for GaN in MOVPE. This method produced very high quality material with FWHM of X-ray rocking curve around 360 arc s. Cao et al (1997, 1998) also used compliant substrates like Si (100) and nitrided the surface before GaN growth. FWHM of Bragg reflection was 366 arc $s$ in this case but the surface was rough and hillocks were also produced. Some other groups also took a similar approach to reduce the stress by nitrogen implantation of a Si (100) substrate (Koh et al 2000) and obtained good results. Therefore, the use of compliant substrates for GaN growth seems to be promising, as the quality of material is comparable to that grown on $\mathrm{SiC}$ or sapphire.

(IV) Patterning substrates by masking or etching the substrates or buffer layer is another low cost but highly effective way to reduce the stress or cracks. Dislocations or cracks will be guided in the masked or etched layer and will leave the epitaxial layer with low density of dislocations or cracks. In this technique, $\mathrm{Si}_{3} \mathrm{~N}_{4}$ or $\mathrm{SiO}_{2}$ layer is deposited over the $\mathrm{Si}$ substrate in a patterned manner or deep trenches are made on the masked materials. Ultimately, lateral epitaxial overgrowth (LEO) takes place and the reports show that thick GaN film can be grown easily (Zamir et al 2001a). The quality of the material is good enough with reference to the PL intensity (Zamir et al 2001b,c). Intensity of PL and FWHM of X-ray rocking curve are comparable to the material grown on $\mathrm{SiC}$ for a field area of $200 \times 200 \mu \mathrm{m}^{2}$ as shown by Honda et al (2002). This approach is promising but suffers from the fact that rather small areas can be grown. However, repeated application of this approach to each LEO layer could produce a large area low defect density GaN layer on $\mathrm{Si}$.

(V) The use of AlGaN buffer layers on AlN seed layer is another interesting way to achieve crack-free thick GaN layers. The AlGaN layer increases the series resistance and generates compressive stresses in the GaN layer, which helps to reduce cracks in the layer and provides good electrical 
insulation from the substrates. So the efficiency of high frequency transistors and vertically contacted LEDs will increase. $1 \mu \mathrm{m}$ thick GaN layer prepared by this technique has shown an X-ray FWHM of 600 arc s and a PL FWHM of $8 \mathrm{meV}$ (Ishigawa et al 1999a,b). The relative brightness of LEDs on GaN on $\mathrm{Si}$ is much higher in this method than in the others. The LED was rated at $20 \mu \mathrm{W}$ at $20 \mathrm{~mA}$ (Egawa et al 2002a).

(VI) A superlattice structure reduces dislocations. This idea can also be applied to growth of $\mathrm{GaN}$ where dislocations tend to bend at the interfaces and they recombine and are some times annihilated by other dislocations. So the superlattice structure of $\mathrm{AlGaN} / \mathrm{GaN}$ can be used to reduce the cracks and dislocations in a thick GaN layer. Also, another advantage is that this superlattice structure can be used as a Bragg reflector in LED structures. A dislocation density 10 times lower than a normal $\mathrm{GaN}$ on $\mathrm{Si}$ sample was achieved by using a 15-fold superlattice structure of AlGaN/GaN having thickness of $0.9 \mu \mathrm{m}$ (Dadgar et al 2001). The top GaN layer in this case yielded $0.35 \mathrm{GPa} / \mu \mathrm{m}$ tensile stress only. AlN/GaN superlattice structures are also used as buffer layers to reduce the dislocations and a crack free $2.5 \mu \mathrm{m}$ thick GaN layer for LED fabrication has been demonstrated (Feltin et al 2001b). Thus, with the developments of these varied approaches to growing $\mathrm{GaN}$ on $\mathrm{Si}$ substrates, the emergence of low cost GaN devices is a matter of time.

\section{Devices}

III-Vs and other group III nitrides have been mainly investigated for their optoelectronic properties. However, the optical properties for these films grown on Si are not good enough and currently only electronic devices are made from $\mathrm{GaN}$ on $\mathrm{Si}$. Waveguide structures and surface acoustic waves (SAW) devices from group III nitrides (Schenk et al 2001) are other research areas of interest. Commercialized GaN FETs and LEDs grown on Si are also now available. Considerable research is being carried out on GaN HEMTs at present.

\subsection{Light emitting diode}

Guha and Bojarczuk (1998a) were the first to make MBE grown GaN LEDs on $\mathrm{Si}$, which demonstrated $\mathrm{Si}$ as an interesting substrate for GaN growth. But the film had several cracks and the efficiency was poor. With the recent developments in this field, along with the advancement in buffer layer or masking or superlattice structures, the efficiency as well as the output power has increased to a level sufficient for commercialization of GaN/Si LEDs. To reduce the absorption of light in the Si substrate, the simplest way is to use wet chemical etching of the substrate and then mounting the epilayers on some metal base. Output power in such structures also increases effectively. Blue LEDs on Si (111) by $\mathrm{InGaN} / \mathrm{GaN}$ multiquantum well in MOVPE was first reported by Tran et al (1999) and the device worked at $4 \mathrm{~V}$. Using $\mathrm{SiO}_{2}$ masking techniques to reduce the cracks, MOVPE was used to produce films with defect density comparable to films on SiC substrates. These LEDs had a turn-on voltage of $3.2 \mathrm{~V}$ (Yang et al 2000) and their series resistance was also smaller than in earlier work. By nitridation of AlAs buffer layers on Si (111) and using a single quantum well it was possible to get bright luminescence (Dadgar et al 2000a) with Pt $p$-type contact and $\mathrm{Al} / \mathrm{Au} n$-type contacts. Thick AlN and AlGaN buffer layers were used by Ishigawa et al (Ishigawa et al 1999b; Egawa et al 2002b) which prevented cracking and the LED power output was $23 \mu \mathrm{W}$ at $20 \mathrm{~mA}$. With the same buffer layer thickness, they increased the thickness of $\mathrm{GaN}$ layer and achieved series resistances as low as 100 ohms. They also investigated vertical contact LEDs (Egawa et al 2002b) with series resistance of around $30 \mathrm{ohm}$. Remarkable work has been done in this regard by Dadgar et al who achieved output power of $155 \mu \mathrm{W}$ at $20 \mathrm{~mA}$. They used low temperature AIN interlayer over 2 " $\mathrm{Si}$ (111) substrate and also applied $\mathrm{Si}_{x} \mathrm{~N}_{y}$ masking on the AlN interlayer (Dadgar et al 2002a). Application of $\mathrm{Si}_{x} \mathrm{~N}_{y}$ and AlN buffer reduced defect densities by almost 10 times (Dadgar et al 2002a,b). Vertically contacted LEDs with $420 \mu \mathrm{W}$ at $20 \mathrm{~mA}$ have been achieved by device structure modification.

Though the work on GaN LEDs on Si has advanced, they are still not comparable to GaN LEDs on $\mathrm{SiC}$ or sapphire. The efficiency as well as the brightness for GaN LEDs on SiC and sapphire is quite high. They can emit $10 \mathrm{~mW}$ at $20 \mathrm{~mA}$ (after packaging), which is still a distant goal for LEDs on Si substrate. But the reported output power for GaN LEDs on $\mathrm{Si}$ are from the labs and not from industry level. Therefore, it may be possible that after epoxy packaging of the LEDs the output power will increase, as in the case of $\mathrm{SiC}$ or sapphire based LEDs. Also, the Si substrate can be removed easily from the above layer, which is not easy for sapphire or SiC; thereby, the absorption due to substrate can be checked. Finally the freestanding layer can be mounted on highly reflective metallic bases, which will act as heat sinks for high brightness LEDs.

\subsection{Transistors}

$\mathrm{GaN}$ on $\mathrm{Si}$ is quite popular for use in HEMT and FET like devices. The background carrier concentration is much lower in $\mathrm{GaN}$ on $\mathrm{Si}$ than on $\mathrm{SiC}$ or sapphire. Also, $\mathrm{Si}$ is a better conductor for heat than sapphire. AlN or Al-rich buffer layers can achieve electrical insulation from the substrate. The most interesting thing in HEMT or FET for $\mathrm{GaN}$ is that a $2 \mathrm{D}$ electron or hole gas forms at the AlGaN/GaN or InGaN/GaN interface without modulation doping. This happens due to spontaneous polarization and 
strain induced piezoelectric fields. Normally the 2Dcarrier sheet density is around $10^{13} / \mathrm{cm}^{2}$ and the mobility is around $1600 \mathrm{~cm}^{2} / \mathrm{Vs}$ at room temperature (Semond et al 2001). The simple FET structure consists of a thin AlN seed or nucleation layer followed by a thick GaN buffer layer. In order to achieve 2D electron or hole gas, a thin AlGaN layer is deposited on top of it (Kaiser et al 2000; Schremer et al 2000). A HEMT having a cutoff frequency of $12.5 \mathrm{GHz}$ and a saturation current of $0.91 \mathrm{~A} / \mathrm{mm}$ (Javorka et al 2002) has been developed on GaN/AlGaN $2 \mathrm{D}$ electron gas where $\mathrm{Si}$ was used as a substrate. The problem with $\mathrm{Si}$ is that it is a poor insulator compared to sapphire and $\mathrm{SiC}$. For better insulation one has to make the buffer layers thicker but this will promote cracking. Therefore, a simpler solution could be to dope the GaN buffer layer with deep levels to increase its insulation property, thereby creating semi-insulating GaN.

\section{Summary}

In the last few years, $\mathrm{GaN}$ growth on $\mathrm{Si}$ has increased rapidly and several solutions are emerging to accommodate the stress due to thermal mismatch. LEDs and FETs are now well developed and expected to be commercialized soon. A blue laser on $\mathrm{Si}$ has been reported by a joint collaboration of RWTH Aachen with AIXTRON AG (Germany) and Stephanov Institute of Physics (Press release: AIXTRON). But its lifetime is not high enough to commercialize it at present. In the near future, Si may be the dominant substrate material for GaN growth due to its low cost and availability in large diameters. The additional advantage is that the $\mathrm{Si}$ substrate can be etched away for laser or LEDs. Also, Si can serve as a substrate to grow GaN template on it by HVPE (Lahrèche et al 2001) for development of GaN substrates.

\section{References}

Amano H, Akasaki I, Hiramatsu K, Koide N and Sawaki N 1988 Thin Solid Films 163415

Amano H, Kito M, Hiramatsu K and Akasaki I 1989 Jpn. J. Appl. Phys. 28 L2121

Amano H et al 1998 Jpn. J. Appl. Phys. 37 L1540

Cao J, Pavlidis D, Eisenbach A, Philippe A, Bru-Chevallier C and Guillot G 1997 Appl. Phys. Lett. 713880

Cao J, Pavlidis D, Park Y, Singh J and Eisenbach A 1998 J. Appl. Phys. 833829

Chen P et al 2001 J. Cryst. Growth 225150

Dadgar A et al 2000a IPAP Conference Series 1845

Dadgar A, Bläsing J, Diez A, Alam A, Heuken M and Krost A 2000b Jpn. J. Appl. Phys. 39 L1183

Dadgar A et al 2001 Appl. Phys. Lett. 782211

Dadgar A, Poschenrieder M, Bläsing J, Fehse K, Diez A and Krost A 2002a Appl. Phys. Lett. 803670

Dadgar A et al 2002b Phys. Status Solidi (a)192 308
Egawa T, Zhang B, Nishikawa N, Ishikawa H, Jimbo T and Umeno M 2002a J. Appl. Phys. 91528

Egawa T, Moku T, Ishikawa H, Ohtsuka K and Jimbo T 2002b Jpn. J. Appl. Phys. 41 L663

Etzkorn E V and Clarke D R 2001 J. Appl. Phys. 891025

Feltin E, Beaumont B, Laügt M, de Mierry P, Vennégués P, Leroux M and Gibart P 2001a Phys. Status Solidi (a)188 531

Feltin E et al 2001b Jpn. J. Appl. Phys. 40 L738

Fu Y, Gulino D A and Higgins R $2000 \mathrm{~J}$. Vac. Sci. Technol. A18 965

Guha S and Bojarczuk N A 1998a Appl. Phys. Lett. 72415

Guha S and Bojarczuk N A 1998b Appl. Phys. Lett. 731487

Hashimoto A, Aiba Y, Motizuki T, Ohkubo M and Yamamoto A 1997 J. Cryst. Growth 175/176 129

Hearne S, Chason E, Han J, Floro J A, Figiel J, Hunter J, Amano H and Tsong I S T 1999 Appl. Phys. Lett. 74356

Honda Y, Kuroiwa Y, Kawaguchi M and Sawaki N 2002 Appl. Phys. Lett. 80222

Ishigawa H, Zhao G Y, Nakada N, Egawa T, Soga T, Jimbo T and Umeno M 1999a Phys. Status Solidi (a)176 599

Ishigawa $\mathrm{H}$, Zhao G Y, Nakada N, Egawa T, Jimbo T and Umeno M 1999b Jpn. J. Appl. Phys. 38 L492

Javorka P, Alam A, Wolter M, Fox A, Marso M, Heuken M, Lüth $\mathrm{H}$ and Kordos P 2002 IEEE Electron. Device Lett. 234

Kaiser S et al 2000 J. Vac. Sci. Technol. B18 733

Koh E K, Park Y J, Kim E K, Park C S, Lee S H, Lee J H and Choh S H 2000 J. Cryst. Growth 218214

Lahréche H, Nataf G, Feltin E, Beaumont B and Gibart P 2001 J. Cryst. Growth 231329

Lee K H, Hong M H, Teker K, Jacob C and Pirouz P 2000 Mater. Res. Soc. Symp. Proc. (Pittsburgh: MRS) Vol. 622

Manasevit H M, Erdmann F M and Simpson W J 1971 J. Electrochem. Soc. 1181864

Marchand H et al 1999 MRS Internet J. Nitride Semicond. Res. 42

Nakamura S, Iwasa N, Senoh M and Mukai T 1992 Jpn. J. Appl. Phys. 311258

Romano L T, Van de Walle C G, Ager III J W, Götz W and Kern R S 2000 J. Appl. Phys. 877745

Schenk H P D, Feltin E, Vaille M, Gibart P, Kunze R, Schmidt H, Weihnacht M and Doghèche E 2001 Phys. Status Solidi (a) 188537

Schremer A T, Smart J A, Wang Y, Ambacher O, Mac Donald N C and Shealy J R 2000 Appl. Phys. Lett. 76736

Semond F, Lorenzini P, Grandjean N and Massies J 2001 Appl. Phys. Lett. 78335

Steckl A J, Devrajan J, Tran C and Stall R A 1996 Appl. Phys. Lett. 692264

Terao S, Iwaya M, Nakamura R, Kamiyana S, Amano H and Akasaki I 2001 Jpn. J. Appl. Phys. 40 L195

Tran C A, Osinski A, Karlicek R F and Berishev I 1999 Appl. Phys. Lett. 751494

Yang J W, Lunev A, Simin G, Chitnis A, Shatalov M, Kahn M A, Van Nostrand J E and Gaska R 2000 Appl. Phys. Lett. 76273

Zamir S, Meyler B and Salzman J 2001a Appl. Phys. Lett. 78 288

Zamir S, Meyler B and Salzman J 2001b J. Cryst. Growth 230 341

Zamir S, Meyler B, Salzman J, Wu F and Golan Y 2001c J. Appl. Phys. Lett. 911191 